\title{
Differentiation of Mesenchymal Stem Cells under Hypoxia and Normoxia: Lipid Profiles Revealed by Time-of-Flight Secondary lon Mass Spectrometry and Multivariate Analysis
}

Citation for published version (APA):

Georgi, N., Cillero-Pastor, B., Eijkel, G. B., Periyasamy, P. C., Kiss, A., van Blitterswijk, C., Post, J. N., Heeren, R. M. A., \& Karperien, M. (2015). Differentiation of Mesenchymal Stem Cells under Hypoxia and Normoxia: Lipid Profiles Revealed by Time-of-Flight Secondary lon Mass Spectrometry and Multivariate Analysis. Analytical Chemistry, 87(7), 3981-3988. https://doi.org/10.1021/acs.analchem.5b00114

Document status and date:

Published: 07/04/2015

DOI:

10.1021/acs.analchem.5b00114

Document Version:

Publisher's PDF, also known as Version of record

Document license:

Taverne

Please check the document version of this publication:

- A submitted manuscript is the version of the article upon submission and before peer-review. There can be important differences between the submitted version and the official published version of record. People interested in the research are advised to contact the author for the final version of the publication, or visit the DOI to the publisher's website.

- The final author version and the galley proof are versions of the publication after peer review.

- The final published version features the final layout of the paper including the volume, issue and page numbers.

Link to publication

\footnotetext{
General rights rights.

- You may freely distribute the URL identifying the publication in the public portal. please follow below link for the End User Agreement:

www.umlib.nl/taverne-license

Take down policy

If you believe that this document breaches copyright please contact us at:

repository@maastrichtuniversity.nl

providing details and we will investigate your claim.
}

Copyright and moral rights for the publications made accessible in the public portal are retained by the authors and/or other copyright owners and it is a condition of accessing publications that users recognise and abide by the legal requirements associated with these

- Users may download and print one copy of any publication from the public portal for the purpose of private study or research.

- You may not further distribute the material or use it for any profit-making activity or commercial gain

If the publication is distributed under the terms of Article 25fa of the Dutch Copyright Act, indicated by the "Taverne" license above, 


\title{
Differentiation of Mesenchymal Stem Cells under Hypoxia and Normoxia: Lipid Profiles Revealed by Time-of-Flight Secondary lon Mass Spectrometry and Multivariate Analysis
}

\author{
Nicole Georgi, ${ }^{\dagger}$ Berta Cillero-Pastor, ${ }^{\dagger, \S}$ Gert B. Eijkel, ${ }^{\ddagger}$ Parthiban C. Periyasamy, ${ }^{\dagger}$ Andras Kiss, ${ }^{\ddagger}$

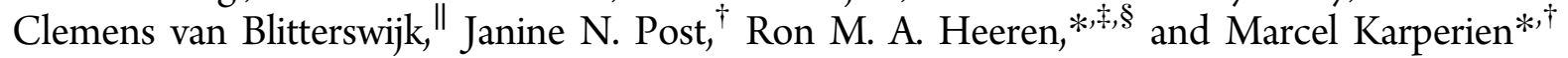 \\ ${ }^{\dagger}$ Developmental BioEngineering, MIRA Institute for Biomedical Technology and Technical Medicine, Faculty of Science and \\ Technology, University of Twente, 7522 NB Enschede, The Netherlands \\ ${ }^{\ddagger}$ Biomolecular Imaging Mass Spectrometry, FOM Institute AMOLF, 1098 XG Amsterdam, The Netherlands

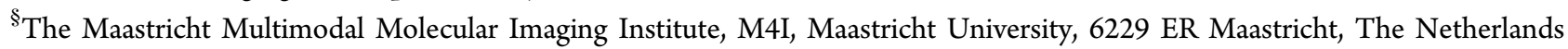 \\ "Department of Tissue Regeneration, MIRA Institute for Biomedical Technology and Technical Medicine, Faculty of Science and \\ Technology, University of Twente, 7522 NB Enschede, The Netherlands
}

Supporting Information

\begin{abstract}
Mesenchymal stem cells (MSC) have the ability to self-renew and differentiate into multiple cell types valuable for clinical treatment of rheumatic pathologies. To study the chondrogenic potential of MSC and identify the conditions that recreate the native cartilage environment, we used time-of-flight secondary ion mass spectrometry (TOF-SIMS) for label-free detection of cell-type- and environmentalcondition-specific molecular profiles. We observed that coculture of human MSC and chondrocytes under standard culture conditions leads to improved extracellular matrix (ECM) deposition. In marked contrast, this effect was lost under low oxygen tension. This improved extracellular matrix deposition was associated with a significant decrease in lipids and in particular cholesterol under low oxygen tension as revealed by TOF-SIMS coupled to principal component analysis and discriminant analysis. We furthermore demonstrate that the higher cholesterol levels under normoxia might regulate fibroblast growth factor 1 (FGF-1) gene expression which was previously implemented in increased ECM production in the cocultures. In conclusion, our study shows an unexpected role of lipids as orchestrators of chondrogenesis in response to oxygen tension which is, at least in part, mediated through FGF-1.
\end{abstract}

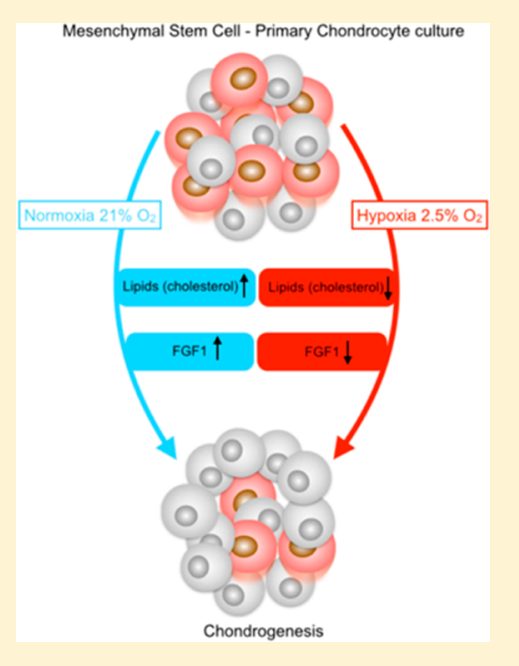

$\mathrm{C}$ artilage is a tissue with low self-repair capability, and therefore it is important to find therapeutic strategies that enable efficient cartilage repair. The current standard for repair of large-size cartilage defects is autologous chondrocyte implantation (ACI). ${ }^{1}$ However, chondrocyte harvest for ACI creates donor site injury and necessitates in vitro expansion of cells to obtain a clinically meaningful population for implantation. Chondrocyte expansion in vitro is associated with dedifferentiation as well as loss of chondrogenic potential. ${ }^{2}$ It has been postulated that partial replacement of chondrocytes with mesenchymal stromal/stem cells (MSCs) can circumvent the problem of expansion and associated loss of function of chondrocytes. We demonstrated that cartilage matrix formation can be improved by the coculture of chondrocytes with $\mathrm{MSCs}^{3,4}$ in vitro. In these cocultures we have identified that fibroblastic growth factor 1 (FGF-1), as a soluble trophic factor produced by MSCs, is necessary to induce improved chondrogenesis. ${ }^{5}$ Until now it remains unclear how FGF-1 expression is regulated under the reduced oxygen level normally present in the joint. Only limited research has been undertaken to understand how hypoxia in the joint environment might influence cellular performance. It has long been known that exposure of human cells to hypoxic conditions can lead to and altered lipid metabolism. ${ }^{6-8}$

Considering the heterogeneity in oxygen concentrations experienced by cartilage in the body we aimed to identify the effect of hypoxia (2.5\% oxygen) in regulating lipid metabolism in chondrocytes and its role in developing articular cartilage.

Differential expression of proteins, lipids, and other components provide unique fingerprints to study retrospectively the environmental conditions to which cells in a tissue are exposed. Time-of-flight secondary ion mass spectrometry imaging (TOF-SIMS) can be employed for the simultaneous visualization of spatial distribution of these variable components in tissue sections according to their mass to charge $(\mathrm{m} / z)$ ratio. ${ }^{9}$ Previous studies identified key membrane molecules

Received: January 9, 2015

Accepted: March 5, 2015

Published: March 5, 2015 
such as phosphocholine and choline as well as cofactors of biological processes, which are indicative of necrotic and apoptotic processes. ${ }^{10,11}$ Recently, the TOF-SIMS approach has been successfully employed for the first time in the cartilage research area to investigate the differences in the molecular composition and lipid distribution between healthy and osteoarthritic cartilage. ${ }^{9}$ In fact, accumulation of fatty acids and other lipids has been found in the superficial area of the diseased cartilage. In addition to this, changes in lipid profiles of MSC monocultures under chondrogenic conditions have been revealed using matrix-assisted laser desorption ionization mass spectrometry imaging (MALDI-MSI) and TOF-SIMS. ${ }^{12}$ Phosphocholine- and sphingomyelin-related species were found in MSC after 2 days of chondrocyte differentiation, while phosphatidylionistols were found as markers of chondrogenesis after 14 days of differentiation.

In the present study we examined whether lipid profiles of chondrogenically differentiating human MSCs, primary chondrocytes, and cocultures of MSCs and primary chondrocytes are dependent on oxygen tension using TOF-SIMS.

We studied cocultures and monocultures of human MSCs and primary chondrocytes under normoxic $\left(21 \% \mathrm{O}_{2}\right)$ and hypoxic conditions $\left(2.5 \% \mathrm{O}_{2}\right)$. We found that the lipid profiles of normoxic and hypoxic cultures are different. We used TOFSIMS for a subsequent side-by-side screen to identify potential lipid mediators of the improved cartilage formation in normoxic cocultures of MSCs and chondrocytes as well as hypoxic chondrocyte cultures. We found cholesterol as one of the most discriminating lipids between cultures with good and reduced chondrogenic performance. Unexpectedly, we found that increased cartilage formation in cocultures of MSCs and primary chondrocytes was lost when the cells were cultured under hypoxia. This loss in cartilage formation was associated with a decrease in the mRNA expression of the chondrogenic marker SOX9 as well as in the expression of FGF-1. This decrease in chondrogenesis was associated with a significant drop in lipids and, in particular, cholesterol. Indeed cholesterol metabolism potently regulated FGF-1 expression in normoxia. Our study reveals an unexpected oxygen tension dependent role of cholesterol as an orchestrator of chondrogenesis in cocultures of MSCs and primary chondrocytes which is at least in part mediated through FGF-1.

\section{EXPERIMENTAL SECTION}

Cell Culture. Human primary chondrocytes (hPCs) were isolated from full-thickness cartilage knee biopsies of patients undergoing total knee replacement. Cartilage was cut and digested as previously described. ${ }^{13}$ Mesenchymal stromal/stem cells were selected by adherence from bone marrow of patients undergoing total hip replacement. For the chondrogenic differentiation experiments three different MSC donors with known differentiation potential were selected. Cocultures of all three MSC donors and hPC monocultures contained the same hPCs donor.

High-density micromass cell pellets were formed by seeding 250000 cells per well in a round-bottom 96-wells plate in chondrocyte proliferation medium (Dulbecco's modified Eagle's medium supplemented with $10 \%$ fetal bovine serum, $1 \times$ nonessential amino acids, $0.2 \mathrm{mM}$ ascorbic acid 2phosphate, $0.4 \mathrm{mM}$ proline, $100 \mathrm{U}$ penicillin, and $100 \mathrm{mg} /$ $\mathrm{mL}$ streptomycin) and centrifuged for $5 \mathrm{~min}$ at $500 \mathrm{rcf}$. Cultures were either kept under normoxic conditions $\left(21 \% \mathrm{O}_{2}\right)$ or hypoxic conditions $\left(2.5 \% \mathrm{O}_{2}\right)$. The medium was refreshed twice a week. MSCs and hPCs were used in following combinations: MSC monocultures (100\% MSCs),; cocultures (80\% MSCs $/ 20 \% \mathrm{hPCs}$ ), and primary chondrocyte monocultures ( $100 \%$ hPCs). ${ }^{4}$ All conditions contained equal cell numbers of 250000 cells per pellet. Pellets for TOF-SIMS experiments, quantitative polymerase chain reaction (qPCR) analysis, and Oil Red $\mathrm{O}$ stain were kept in culture for 7 days to detect early changes in lipid levels. Pellet for chondrogenic matrix staining were kept in culture for 28 days. To block the availability of free cholesterol in the cytoplasm U18666a was used at a concentration of $3 \mu \mathrm{g} / \mathrm{mL}$.

Histology: Alcian Blue Staining. Pellets were fixed with $10 \%$ formalin in PBS for 15 min, dehydrated, and embedded in paraffin using routine procedures. Sections of $5 \mu \mathrm{m}$ were cut and stained for sulfated glycosaminoglycans (GAGs) with alcian blue $\left(0.5 \%\right.$, in $\mathrm{H}_{2} \mathrm{O}, \mathrm{pH}=1$ adjusted with $\left.\mathrm{HCl}, 30 \mathrm{~min}\right)$ combined with counterstaining of nuclear fast red $(0.1 \%$ in $5 \%$ aluminum sulfate, $5 \mathrm{~min}$ ).

Lipid Staining. Cryosections of pellets were hydrated in distilled water for $5 \mathrm{~min}$, dipped in $60 \%$ isopropyl alcohol, and then stained with $0.03 \%$ Oil Red solution (60\% isopropyl alcohol) for $20 \mathrm{~min}$. After rinsing in water, the pellet sections were counterstained with hematoxylin for 5 min (SigmaAldrich), washed, dehydrated, and mounted. Images were acquired with the MIRAX imaging system (Carl Zeiss). Lipid droplet accumulation was quantified using color range tool in Adobe Photoshop software as described previously ${ }^{14}$ and tested for statistical significance (ANOVA test, Bonferroni posthoc test, $p<0.01)$.

RNA Isolation and Quantitative PCR. After 7 days of culture, RNA from cell pellets $(n=3)$ cultured under different conditions was pooled and washed with PBS. Trizol reagent (Invitrogen) was used to lyse the pellets. Total RNA was isolated with the standard Trizol RNA isolation protocol with an additional $\mathrm{O} / \mathrm{N}$ RNA precipitation step in $0.3 \mathrm{M}$ sodium acetate and $15 \mu \mathrm{g} / \mathrm{mL}$ linear acrylamide. RNA pellets were airdried and resuspended in ultrapure water. RNA concentration was measured using Nanodrop 2000 (ND-1000 spectrophotometer, Isogen Lifescience).

The RNA samples were treated with DNase I (Invitrogen) following manufacturers protocol. Equal amounts of RNA from samples were used for cDNA synthesis using iScript kit (BioRad) according to the manufacturers protocol. Amplification of cDNA was done using Sensimix SYBR (Bioline) in a CFX Connect Real-Time PCR detection system (Biorad). The protocol for amplification is denaturation at $95{ }^{\circ} \mathrm{C}$ for $10 \mathrm{~min}$, 44 cycles of $95^{\circ} \mathrm{C}$ for $15 \mathrm{~s}, 60^{\circ} \mathrm{C}$ for $15 \mathrm{~s}, 72^{\circ} \mathrm{C}$ for $15 \mathrm{~s}$, and melt curve of $55-95{ }^{\circ} \mathrm{C}$ with $0.5{ }^{\circ} \mathrm{C}$ increment for 5 s per step. The relative expression of each gene was done using delta delta Ct method. ${ }^{15}$ RPL13 was used for normalization. ${ }^{16}$ The primer sequences are as follows: SOX9-Fw, 5'-TGGGCAAGCTCTGGAGACTTC-3'; SOX9-Rev, 5'-ATCCGGGTGGTCCTTCTTGTG-3'; FGF-1-Fw， 5'-AGCCGGGCTACTCTGAGAAGAAGA-3'; FGF-1-Rev, 5'-CTGCTGCTTGTGGCGCTTTCA-3'; RPL13-Fw, 5'-AAAAAGCGGATGGTGGTTC-3'; RPL13-Rev, 5'-CTTCCGGTAGTGGATCTTGG-3'

TOF-SIMS Experiments. High-density micromass cultures of three MSC donors cultured under hypoxic and normoxic conditions were embedded in tissue arrays using $10 \%$ gelatin (embedded at $30{ }^{\circ} \mathrm{C}, 4 \mathrm{~h}$ at $4{ }^{\circ} \mathrm{C}$, frozen at $-20{ }^{\circ} \mathrm{C}$ ) (Supporting Information Figure 1). Cryosections $(20 \mu \mathrm{m}$ thick) of both tissue arrays were mounted side by side on an indium-tin oxide coated conductive glass slide (ITO; $4-8 \Omega$ 

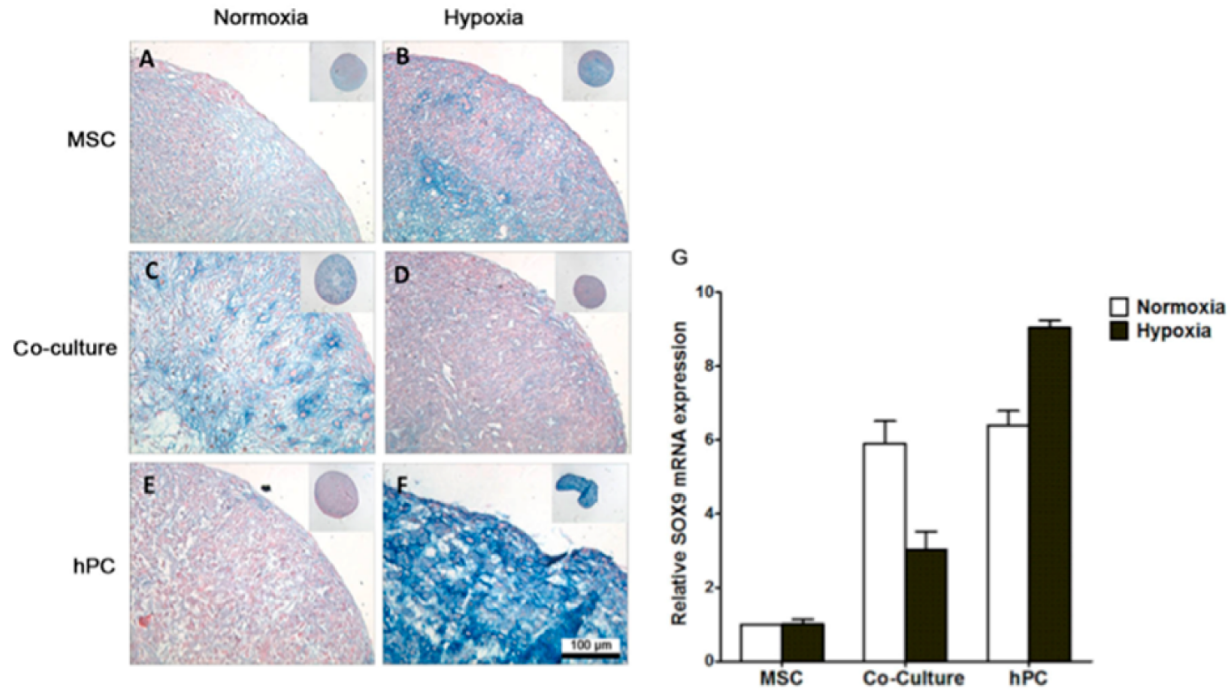

Figure 1. Alcian blue staining of glycosaminoglycans in micromass pellet cultures expanded under normoxia $\left(21 \% \mathrm{O}_{2}\right)$ and hypoxia $\left.2.5 \% \mathrm{O}_{2}\right)$. $(\mathrm{A}, \mathrm{C}$, and E) Pellets expanded in normoxia; (B, D, and F) pellets expanded in hypoxia; coculture of MSCs and chondrocytes which were cultured in normoxia (B) reveal an improved chondrogenesis marked by more intense alcian blue stain; the same coculture in hypoxia (D) results in a reduction of alcian blue staining; chondrocyte monoculture pellets cultured in normoxia (E) exhibit significant less alcian blue staining when compared to chondrocytes in cultured in hypoxia (F). (G) Relative expression of SOX9 in cell pellets cultured in hypoxia or normoxia: in coculture an increased expression of SOX9 is observed when cultured in normoxia. hPCs cultured in hypoxia express the highest SOX9 mRNA levels. $n=3$ technical replicates of three pooled pellets.

resistance, Delta Technologies, Stillwater, MN, U.S.A.), washed with $100 \%$ ethanol (30 s), and $70 \%$ Ethanol $(2 \mathrm{~min})$. This allowed for sampling of 18 sections of pellet cultures simultaneously and reduced intrameasurement variations. Samples were vacuum-dried and coated with $2 \mathrm{~nm}$ of gold (SC7640 sputter coater, FT707 quartz crystal microbalance stage) for TOF-SIMS measurements. TOF-SIMS experiments were performed in positive ion mode on a Physical Electronics (Eden Prairie, MN) TRIFTII secondary ion mass spectrometer with a $\mathrm{Au}^{1+}$ primary ion beam. For the experiments a primary ion beam of $7.2 \mathrm{nA}$, a primary ion pulse length of $19 \mathrm{~ns}$, and an ion dose of $2.14 \times 10^{11}$ ions $/ \mathrm{cm}^{2}$ were used. Whole pellet sections were analyzed in mosaic mode $(64 \times 64$ tiles $)$ with an acquisition time of $3 \mathrm{~s} /$ tile $(256 \times 256$ pixels/tile $)$. The raster size was set at $110 \mu \mathrm{m}$ yielding $426 \mathrm{~nm} /$ pixel. WinCadence 4.4 software was used for data acquisition.

Data Analysis. Raw data was converted and analyzed with our in-house build ChemomeTricks toolbox for MATLAB 2011 b (MathWorks, Inc., Natick, MA, U.S.A.). Spatial binning to $128 \times 128$ pixels, spectral binning to $0.1 \mathrm{amu}$, and peak picking were performed. A total of $1366 \mathrm{~m} / z$ peaks in the range of 1-2000 amu were picked based on $S / N>3$.

Linear discriminant analysis (LDA) was used for determining the specific mass channels that were associated with the different conditions. ${ }^{17}$ For TOF-SIMS measurements sections of the pellets were selected as regions of interest (ROI), and a total of 13198 spectra were used for analysis. LDA revealed the most discriminating masses: (a) in a global analysis of all hypoxic and all normoxic cultured conditions (six groups), (b) in an intercondition (normoxia only and hypoxia only) analysis of different conditions in hypoxia as well as normoxia (2 times three groups), and (c) comparison of specific conditions cultured in hypoxia and normoxia (two groups).

The distributions of the scores for different discriminant functions (DFs) of comparison (b) were calculated. These distributions and their overlap describe the sample identity and were used to identify differences between conditions. The mass channels corresponding to previously classified lipids were identified and assigned as determined by lipid $\mathrm{m} / \mathrm{z}$ identifications using the database on lipidmaps.org (http:// www.lipidmaps.org/) and according literature. ${ }^{18}$

\section{RESULTS}

Coculture Leads to Improved Chondrogenesis under Normoxia $\left(21 \% \mathrm{O}_{2}\right)$. In order to investigate the chondrogenic potential of MSC and chondrocyte mono- and cocultures in normoxia and hypoxia, histological sections were evaluated for glycosaminoglycan deposition and cell morphology after 28 days of culture.

In accordance with the literature, ${ }^{19}$ cartilage formation of chondrocyte monocultures and to lesser extent of MSCs monocultures under hypoxia was improved as compared to pellet cultures in normoxia (Figure 1). Alcian blue staining was used to evaluate the glycosaminoglycan deposition and as an indication of cartilage formation. Improved chondrogenesis was marked by more intense alcian blue staining which coincided with increased SOX9 mRNA expression (Figure 1G). In line with previous observations, ${ }^{4}$ normoxic cocultures of MSCs and chondrocytes demonstrated improved chondrogenesis when compared to respective monocultures (compare Figure 1, part C with parts $A$ and E). In marked contrast with pellet cocultures differentiated in normoxia, hypoxic coculture pellets did not reveal evidence for improved cartilage formation (compare Figure 1, parts D and F). This unexpected observation is supported by the lower expression of the chondrogenic marker SOX9 in hypoxic cocultures as compared to the expression in the normoxic cocultures (Figure 1G).

Normoxic Culture Conditions Result in More Lipid Deposition. In order to elucidate differences in lipid content in pellets cultured under hypoxic and normoxic conditions we first performed an Oil Red O lipid staining which stains neutral triglycerides and lipoproteins. 
Oil Red O staining revealed a significantly higher abundance of lipid droplets in normoxic conditions (Figure 2A).

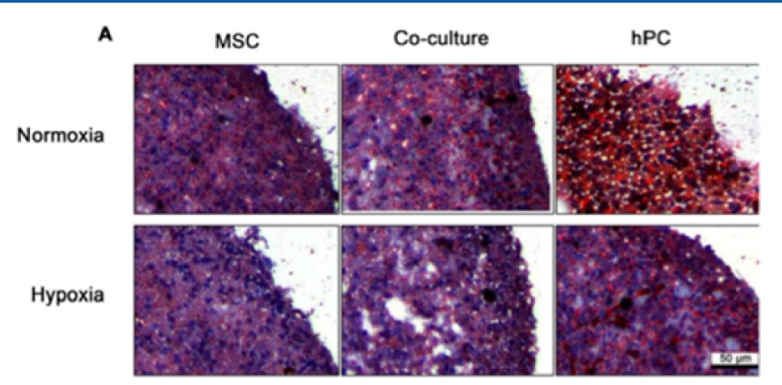

B

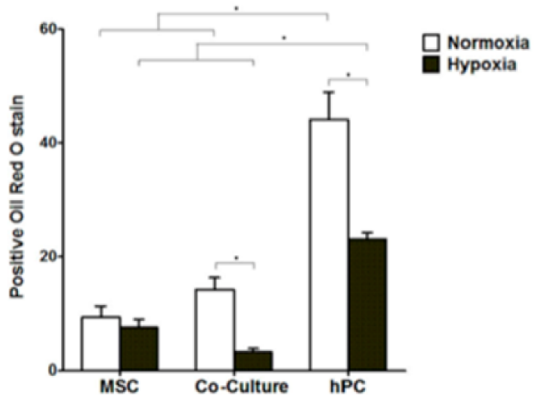

Figure 2. Oil Red $\mathrm{O}$ stain for lipid deposition of pellets cultured in hypoxic and normoxic conditions. (A) Oil Red O stain revealed a higher staining in normoxic conditions. In particular, chondrocyte pellets showed the highest neutral lipid accumulation. (B) Quantification of Oil Red O stain as percentage of the total cell pellet showed that normoxic pellets had a higher lipid content with highest concentration in chondrocyte pellets followed by the coculture pellets; in hypoxia lipid concentration was still highest in the chondrocyte pellets but lowest in the coculture pellets, ${ }^{*} p<0.05$.

Quantification of Oil Red O stain demonstrated that all cellular conditions have a significantly higher lipid concentration in normoxia. Remarkably, chondrocyte pellets cultured in normoxia contained the highest concentration of neutral lipids. The lipid concentration dropped significantly when chondrocytes were cultured in hypoxia. This trend was also seen in the cocultures, where the amount of lipids was greatly reduced when cultured in hypoxia as compared to normoxia (Figure 2B). Since the changes in lipid profiles correlated with the loss of chondrogenic potential of cocultured MSCs and chondrocytes (Figures 1 and 2), we postulated that lipids and/or lipid metabolism might play a role in chondrogenesis. ${ }^{4}$

Molecular Profile of Normoxic Pellet Cultures Separates Clearly from That of Hypoxic Pellet Cultures. Since we wanted to further examine the role of lipids in chondrogenesis, we applied TOF-SIMS to reveal changes in the content of specific lipids and as a method to distinguish between states of differentiation based on these lipid profiles. TOF-SIMS has the advantage of detecting thousands of different molecular species in a single experiment while preserving information on their spatial distribution. A tissue array was employed to study the differences between hypoxia and normoxia by TOF-SIMS (Supporting Information Figure 1). Measurements from all conditions were analyzed by principal component analysis (PCA), and 50 principal components (PCs) explaining 50\% of the variance were used for the LDA. As illustrated in the scatter plot in Figure 3A, the first discriminant function (DF1) separates the MSCs and cocultures from chondrocytes irrespective of the oxygen tension: monocultured chondrocytes (red and black fraction) in the negative part of the graph and monocultured MSCs and cocultures in the positive part. The second DF distinguishes pellets cultured in normoxia (red, green, and blue fraction) from pellets cultured in hypoxia (black, purple, aquamarine) (hypoxia: DF2+, normoxia: DF2-) (Figure 3A). This indicates that the lipid composition of pellets is dependent on the cell as well as the oxygen tension to which they were exposed and thus can be used as a retrospective fingerprint to determine the previous exposure to an environmental condition. Furthermore, we demonstrate that spectra of pellets cultured under normoxia are spreading more among the DF1 than pellets cultured under hypoxia. In respect to the discriminating mass channels this indicates that cells, either being primary chondrocytes or MSCs, are less alike under normoxia than under hypoxia (Figure 3A). This reveals that the different cell types present a more similar molecular profile under hypoxic conditions. The discriminant loading spectra for Figure 3A display the separation of specific masses according to the positive and negative DF2 (Figure 3B). The positive fraction of masses indicates the specific molecular profile under hypoxic culture, whereas the negative side indicates normoxia specific peaks. On the negative side of the plot, displaying the profile of all conditions cultured in normoxia we can observe specific cholesterol masses in the spectra $(\mathrm{m} / z$ 369.3 $[\mathrm{M}-$ $\mathrm{OH}]^{+}, m / z 385.3[\mathrm{M}-\mathrm{H}]^{+}$, and $\left.m / z 970[2 \mathrm{M}+\mathrm{Au}]^{+}\right)$, which were identified as major discriminating masses from the positive hypoxia specific fraction. Furthermore, the higher abundance of mass peaks in the range of $500-2000 \mathrm{~m} / z$ in normoxic conditions indicates that cells cultured in normoxia contain a higher lipid content. These findings are in agreement with the results of the Oil Red $\mathrm{O}$ staining for lipids in cells cultured under these conditions. On comparing spectra of coculture pellets from hypoxic and normoxic conditions, cholesterol peaks are found to be the most separating masses. Other differentially expressed peaks correspond to masses tentatively assigned to diacylglycerols (Figure 3D). Discriminant score frequency plots of coculture pellets in hypoxic and normoxic conditions were clearly distinct. This suggests that the oxygen concentration in culture conditions strongly influences the lipid composition present in cells (Figure 3C).

Figure 4 represents the reconstructed score image of DF2 spectra of MSCs, coculture, and hPCs of the LDA that is depicted in Figure 3A. The negative scores (representing lipid changes) can be observed in normoxia, whereas the modulation of lipid expression observed in hypoxia was more limited. All three used MSC donors display the same trend regarding their corresponding lipid profile, whereas donor variation in absolute values is visible. For instance, donor three presented a higher contrast between the three cell combinations and at the same time the highest lipid levels. This underlines the power of TOFSIMS to discriminate between the specific molecular signatures of different cell culture conditions as well as different donors, which would be crucial for the selection of the best combination of environmental factors and the cell source/ donor for a successful chondrocyte differentiation.

High Oxygen Concentrations Cause More Differences in Lipid Profiles than Hypoxia. By separate LDA of the cells cultured in two different oxygen concentrations we obtained insight in differences between these cellular conditions. The displayed DF1 in Figure 5, parts A and B, differentiate lipid signatures of monoculture and coculture pellets of MSCs and hPCs. Remarkably, the groups are less defined and the separation is less clear in hypoxia (Figure 5B) when compared 

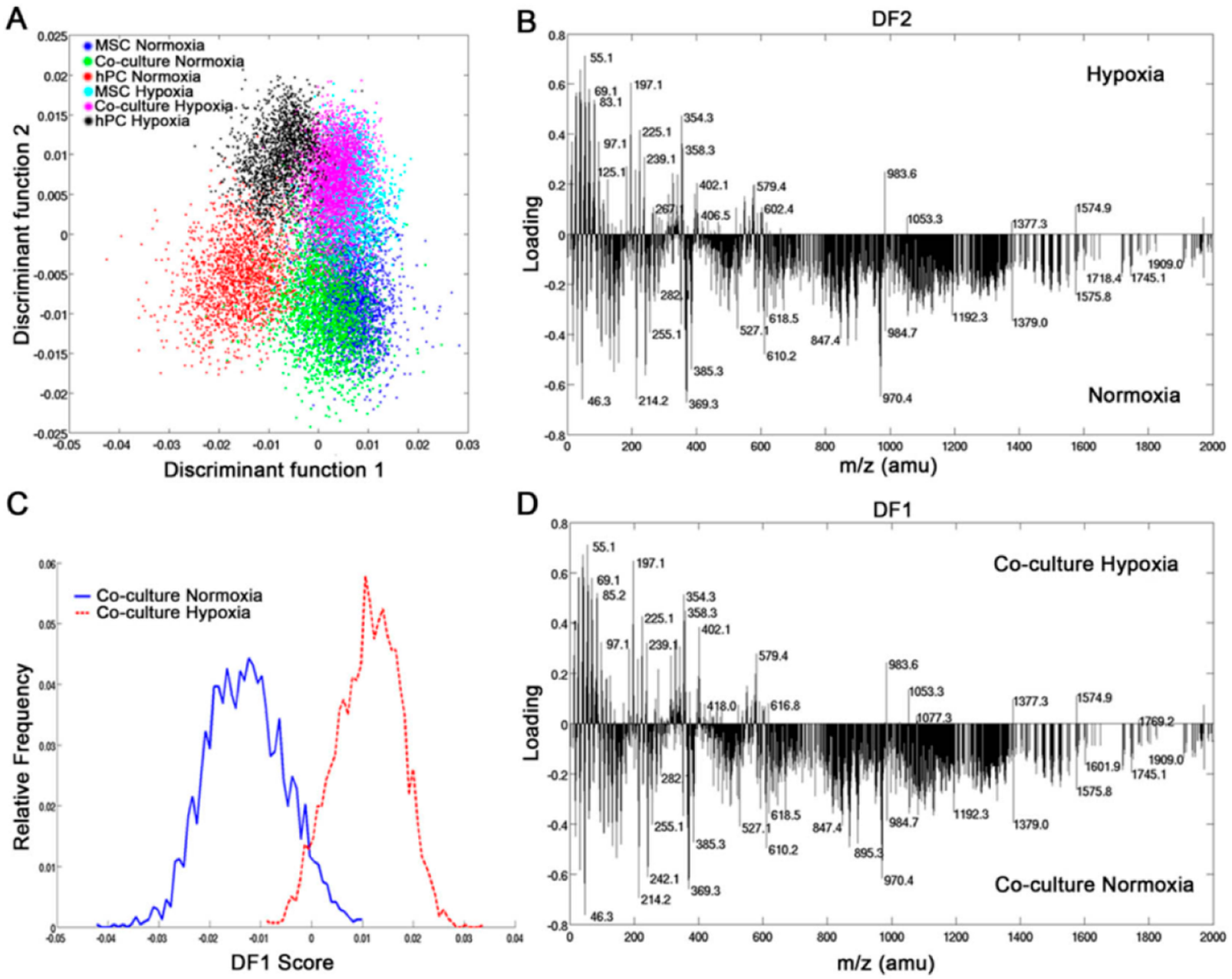

Figure 3. Discriminant analysis of cells in hypoxic and normoxic culture conditions. (A) Tissue arrays of pellet cultures in normoxia and hypoxia were mounted on the same slide and analyzed using TOF-SIMS. Spectra of all pellets were obtained and analyzed using PCA. Fifty principal components (PCs) (50\% of the variance) were used for the discriminant function (DF) analysis: hypoxic and normoxic conditions separate among the second DF (hypoxia $>0$, normoxia $<0$ ); normoxia is spreading more among the first DF indicating that different conditions are less alike than in hypoxia. (B) The according discriminant loading spectra for panel A showing specific cholesterol masses $\left(\mathrm{m} / z 369.3[\mathrm{M}-\mathrm{OH}]^{+}, \mathrm{m} / z 385.3[\mathrm{M}-\right.$ $\mathrm{H}]^{+}$, and $m / z 970[2 \mathrm{M}+\mathrm{Au}]^{+}$) for the normoxic cultured conditions. (C) Frequency plots of the hypoxia and normoxia cultured coculture display a clear separation of both conditions, (D) comparing only coculture conditions of both oxygen tensions reveals cholesterol still as one of the most separating masses between hypoxia and normoxia.

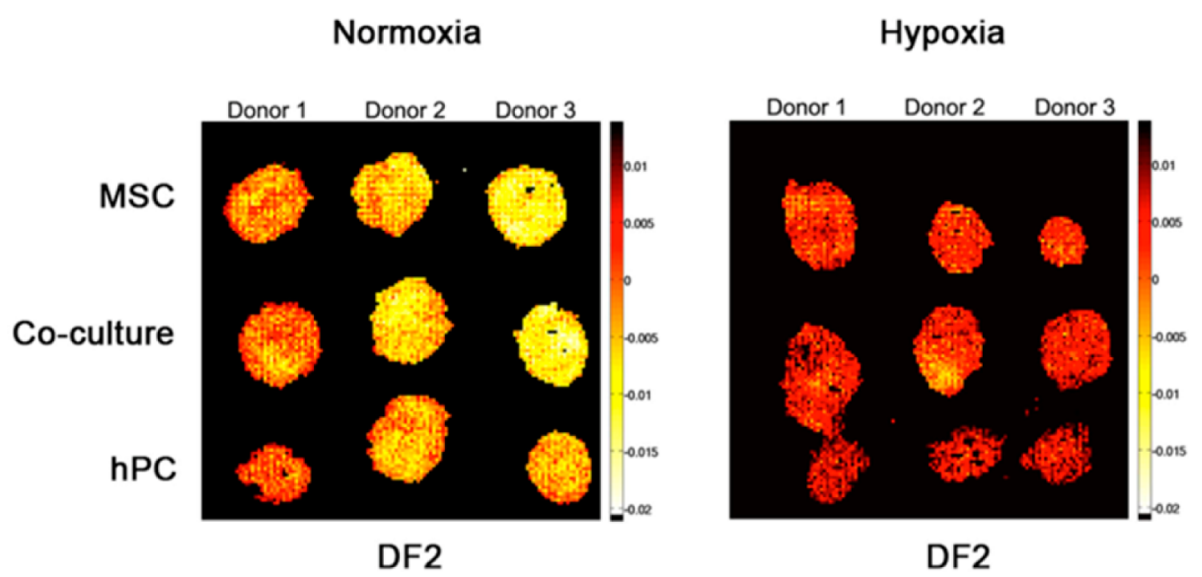

Figure 4. Reconstructed score image of DF2. Image represents the projection of the DF2 spectra of MSCs, coculture, and hPCs in hypoxic or normoxic conditions. Overall differences in molecular profiles between normoxia and hypoxia are depicted underlining the high presence of lipids under normoxia. 

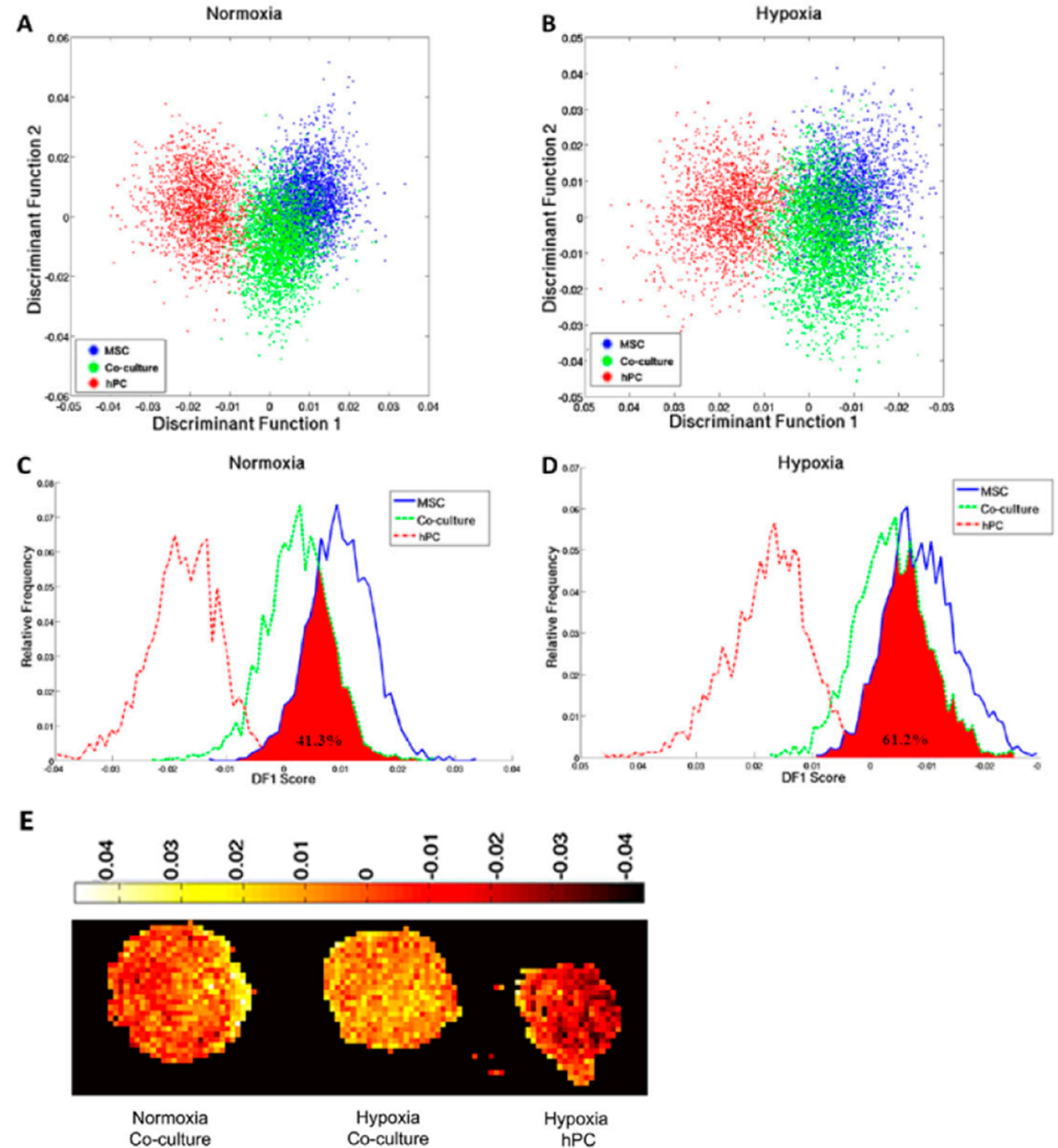

Figure 5. Micromass cultures display distinct molecular profiles in hypoxia and normoxia. (A and B) Discriminant function analysis of the cocultured and monocultured cell pellets in normoxic and hypoxic culture: in normoxia (A) MSC monocultures (100\% MSCs) and the cocultures ( $80 \%$ MSCs/ $20 \% \mathrm{hPCs}$ ) partially separate among the first DF, whereas there is no separation between these conditions in hypoxia in the first discriminant function (B). (C and D) Respective frequency plots of the DF1 shown in panels A and B underlining the better separation of the cocultures from MSC monocultures in normoxia (C). (E) The reconstructed score image of DF2 shows that the coculture in normoxia presents a more similar molecular profile to hPCs in hypoxia than the coculture condition in hypoxia.

to normoxia (Figure 5A). Normoxic cocultures display a $41.3 \%$ of identity with MSC monocultures (Figure 5C), whereas hypoxic cocultures show a $61.2 \%$ overlap with MSC monocultures (Figure 5D). We already demonstrated that MSCs have a stimulatory role over chondrocytes under normoxia $^{5}$ and that only a minor fraction of the initial $80 \%$ MSCs is left in cocultures under normoxia. ${ }^{4}$ The higher identity overlap of cocultures with pure MSC pellet cultures in hypoxia indicates that the mechanisms underlying the stimulatory role of MSCs on chondrocyte proliferation and chondrogenesis, as described previously, ${ }^{5}$ are delayed or less abundant in hypoxia.

In the discriminant analysis of all conditions (six groups) cholesterol was detected as a lipid that was highly enriched in pellets cultured in normoxia. Resulting from the separate discriminant analysis of pellets in normoxic conditions (three groups, Figure 5A) a discriminant-loading spectrum was generated (Supporting Information Figure 2). On the basis of the mass separation among the first DF the identification of two groups was possible: MSCs grouped together with cocultures versus hPCs monocultures. In chondrocytes phosphocholine, $m / z$ 184.1, was increased, whereas in cultures containing MSCs diacylglycerol, $m / z$ 577.4, $m / z$ 603.4, and cholesterol, $m / z$ 369.3, were detected.

Alcian blue staining and SOX9 mRNA expression underlined that the ideal conditions for matrix formation of chondrocyte monocultures are provided under hypoxia, whereas MSC containing cocultures display better matrix formation under normoxia. We performed LDA in order to observe these molecular changes that mark a better chondrogenic performance in respective conditions. For this we compared hPCs under hypoxia and cocultures under hypoxia and normoxia. We analyzed each donor separately. The DF1 separated cultures grown under normoxic and hypoxic conditions (not shown). The DF2 displayed that the cocultures in normoxia had a more similar molecular distribution to chondrocytes in hypoxia than the coculture in hypoxia (Supporting Information Figure 3). In 
line with these findings Figure 5E represents the DF2 score image of the samples shown in Supporting Information Figure 3. The coculture in normoxia was more similar to the chondrocyte molecular profile in hypoxia. This again supports the finding that the improved chondrogenesis in cocultures functions under normoxic culture conditions but is hampered under hypoxia. We believe that this change in performance can be linked to changes in lipid expression in response to oxygen tension.

Cholesterol Inhibition Alters FGF-1 Expression at the mRNA Level. In our previous studies we have demonstrated that, during normoxic coculture of MSCs and hPCs, FGF-1 is secreted by MSCs and that this positively influences the proliferation and chondrogenic performance of chondrocytes. ${ }^{20}$ Since cell-cell communication and expression of specific receptors localized in the cell membrane depend on their lipid constitution, we compared FGF-1 expression in hypoxic and normoxic culture conditions. We found that the FGF-1 mRNA expression is higher in normoxic cocultures as compared to hypoxic cocultures. These results correlate with the alcian blue staining, and SOX9 results that show a better chondrogenic performance of the coculture under normoxia as compared to hypoxia. Under hypoxic conditions MSCs produce less FGF-1 and chondrocytes produce more FGF-1 as compared to their normoxic counterparts (Figure 6).

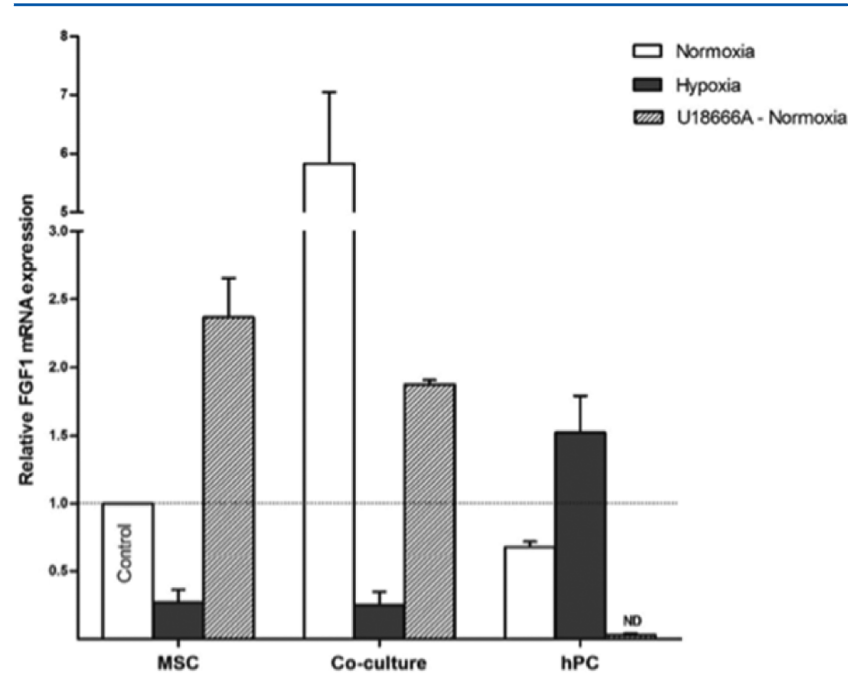

Figure 6. Expression of FGF-1 mRNA in co- and monopellet cultures under normoxia and hypoxia. FGF-1 expression changes dramatically in MSCs and hPCs when free cholesterol is blocked in normoxic conditions. $n=3$ technical replicates of pellets from three donors that are pooled. Expression is measured relative to the expression of the RPL13 gene.

To test whether lipids, such as cholesterol, have any role in regulating the FGF-1 mRNA expression, we blocked the cholesterol pathway using U18666a under normoxia. In the presence of U18666a, cells accumulate free cholesterol in the lysosomes, resulting in depletion of free cholesterol from the cytoplasm. $^{21}$

When free cholesterol is blocked in pellets cultured under normoxia a noticeable change in FGF-1 mRNA levels is seen. FGF-1 mRNA levels decreased in the coculture showing the relation between FGF-1 expression and cholesterol availability. MSCs respond to low cholesterol by up-regulating FGF-1 mRNA expression. On the other hand, chondrocytes respond to low cholesterol by blocking FGF-1 mRNA expression (Figure 6). We have previously shown that the FGF-1 gene expression in cocultures under normoxia is mainly contributed by MSCs. ${ }^{5}$ However, in hypoxic conditions, FGF-1 expression is linked to the lower cholesterol levels in both MSCs and hPCs. This FGF-1 expression dependency on cholesterol levels has not been reported so far.

\section{DISCUSSION}

With the use of TOF-SIMS as a tool for label-free detection of molecular profiles specific to different cell types and exposure to environmental conditions, we demonstrate that the molecular fingerprints of human MSCs, primary chondrocytes, and MSC/primary chondrocytes coculture differ when cultured in either normoxic $\left(21 \% \mathrm{O}_{2}\right)$ or hypoxic $\left(2.5 \% \mathrm{O}_{2}\right)$ conditions. Under hypoxic conditions we observed a loss of the chondrogenic inductive effect of MSCs. This coincided with a significant decrease in lipids. In addition we observed a sharp decrease in FGF-1 mRNA production, which was at least in part regulated by the cholesterol concentration in the cells. Cholesterol and other lipids play a major role in providing cell membrane integrity as well as play a pivotal role in cellular signaling and metabolism. Thus, lipid levels in the cell might be crucial for mediating cellular responses, including differentiation, proliferation, and ultimately chondrogenesis in cocultures of MSCs and primary chondrocytes. ${ }^{22}$

Conventional approaches to study lipids in cells mostly do not allow for a detailed observation of changes in a broad spectrum of lipid molecules. As of yet, this is the first report to describe the differences in lipid profiles by using a highthroughput technology such as TOF-SIMS and provides compelling evidence that this technique can be used to characterize environmental conditions to which cells previously have been exposed. It is important to perform complementary analysis with other methods that confirm that the found differences are not due to artifacts, sensitivity issues, and due to the heterogeneity of the molecular environment of the samples. Therefore, we have shown in this study that TOF-SIMS has an important potential to identify the molecular profiles specific to different donors and their correlation with the stage of cell differentiation. Previously, we have also demonstrated by MALDI-MSI and TOF-SIMS that there is a different lipid profile in MSC monocultures after 2 and 14 days of chondrogenic differentiation. ${ }^{12}$

It has been hypothesized that the phenomenon of improved cartilage formation in cocultures of MSCs and chondrocytes is dependent on soluble factors, including small molecules, lipids, or proteins. ${ }^{23}$ Lipids such as phospholipids, cholesterol, and diacylglycerols play significant roles in cellular signaling, membrane integrity, and metabolism. ${ }^{22}$, Recently, Simonaro et al. described that short-term changes in sphingolipid metabolism may lead to long-term effects on the chondrogenic phenotype and that the stimulation of chondrocytes with acyl ceramidase improves cartilage repair and MSC differentiation. ${ }^{24}$

Our previous research showed that during coculture of MSC and chondrocytes under normoxia FGF-1, fibroblast growth factor 1 , is produced by MSCs as a trophic factor influencing chondrocyte proliferation and chondrogenic performance. ${ }^{20}$ In this study we see that hypoxia and normoxia elicit distinct effects on FGF-1 mRNA expression. Our study indicates that changes in cholesterol levels affect the production of FGF-1 that promotes cartilage formation. The loss of coculture effect in hypoxia could be explained at least in part by a significant 
drop in FGF-1 signaling. This drop might be caused by a decrease in cholesterol.

\section{CONCLUSIONS}

Here we successfully used for the first time TOF-SIMS in combination with multivariate analysis to define differences in lipid profiles between MSCs, primary chondrocytes, and cocultures under different oxygen tensions. This approach enables us to identify the key signaling pathways that improve stem cell differentiation to cartilage and their potential application for cell therapy strategies.

\section{ASSOCIATED CONTENT}

\section{S Supporting Information}

Additional information as noted in text. This material is available free of charge via the Internet at http://pubs.acs.org.

\section{AUTHOR INFORMATION}

\section{Corresponding Authors}

*Phone: 0031-627245427.E-mail: r.heeren@ maastrichtuniversity.nl.

*Phone: 0031-53-489-3323/3400. Fax: 0031-53-489-2150. Email: h.b.j.karperien@utwente.nl.

\section{Author Contributions}

N.G. and B.C.-P. contributed equally to this work.

\section{Notes}

The authors declare no competing financial interest.

\section{ACKNOWLEDGMENTS}

The authors gratefully acknowledge the support of the TeRM Smart Mix Program of The Netherlands Ministry of Economic Affairs and The Netherlands Ministry of Education, Culture and Science to N.G., C.v.B., and M.K. and an unrestricted program subsidy of the Dutch Arthritis Association to M.K. B.C.-P. is supported by the Dutch national program COMMIT.

\section{REFERENCES}

(1) Brittberg, M.; Lindahl, A.; Nilsson, A.; Ohlsson, C.; Isaksson, O.; Peterson, L. N. Engl. J. Med. 1994, 331, 889-895.

(2) Ma, B.; Leijten, J. C.; Wu, L.; Kip, M.; van Blitterswijk, C. A.; Post, J. N.; Karperien, M. Osteoarthritis and Cartilage 2013, 21, 599603.

(3) Hendriks, J.; Miclea, R.; Schotel, R.; de Bruijn, E.; Moroni, L.; Karperien, M.; Riesle, J.; van Blitterswijk, C. Soft Matter 2010, 6, 5080-5088.

(4) Wu, L.; Leijten, J. C.; Georgi, N.; Post, J. N.; van Blitterswijk, C. A.; Karperien, M. Tissue Eng., Part A 2011, 17, 1425-1436.

(5) Wu, L.; Leijten, J.; van Blitterswijk, C. A.; Karperien, M. Stem Cells Dev. 2013, 22, 2356-2367.

(6) Bostrom, P.; Magnusson, B.; Svensson, P. A.; Wiklund, O.; Boren, J.; Carlsson, L. M.; Stahlman, M.; Olofsson, S. O.; Hulten, L. M. Arterioscler., Thromb., Vasc. Biol. 2006, 26, 1871-1876.

(7) Gimm, T.; Wiese, M.; Teschemacher, B.; Deggerich, A.; Schodel, J.; Knaup, K. X.; Hackenbeck, T.; Hellerbrand, C.; Amann, K.; Wiesener, M. S.; Honing, S.; Eckardt, K. U.; Warnecke, C. FASEB J. 2010, 24, 4443-4458.

(8) Libby, P.; Folco, E. Circ. Res. 2011, 109, 1100-1102.

(9) Cillero-Pastor, B.; Eijkel, G.; Kiss, A.; Blanco, F. J.; Heeren, R. M. Anal. Chem. 2012, 84, 8909-8916.

(10) Fletcher, J. S. Analyst 2009, 134, 2204-2215.

(11) Amstalden van Hove, E. R.; Blackwell, T. R.; Klinkert, I.; Eijkel, G. B.; Heeren, R. M.; Glunde, K. Cancer Res. 2010, 70, 9012-9021.
(12) Rocha, B.; Cillero-Pastor, B.; Eijkel, G.; Bruinen, A. L.; RuizRomero, C.; Heeren, R. M.; Blanco, F. J. Proteomics 2015, 15, 702713.

(13) Hendriks, J.; Riesle, J.; Vanblitterswijk, C. A. Tissue Eng. 2006, 12, 2397-2405.

(14) Tolivia, J.; Navarro, A.; del Valle, E.; Perez, C.; Ordonez, C.; Martinez, E. Anal. Quant. Cytol. Histol. 2006, 28, 43-53.

(15) Livak, K. J.; Schmittgen, T. D. Methods 2001, 25, 402-408.

(16) Pombo-Suarez, M.; Calaza, M.; Gomez-Reino, J. J.; Gonzalez, A. BMC Mol. Biol. 2008, 9, 17.

(17) Hoogerbrugge, R.; Willig, S. J.; Kistemaker, P. G. Anal. Chem. 1983, 55, 1710-1712.

(18) Passarelli, M. K.; Winograd, N. Biochim. Biophys. Acta 2011, 1811, 976-990.

(19) Lawyer, T. J.; Tucci, M. A.; Benghuzzi, H. A. Biomed. Sci. Instrum. 2012, 48, 246-253.

(20) Wu, L.; Leijten, J.; van Blitterswijk, C. A.; Karperien, M. Stem Cells Dev. 2013, 22, 2356-2367.

(21) Liscum, L.; Faust, J. R. J. Biol. Chem. 1989, 264, 11796-11806.

(22) Brown, A. J. Clin. Exp. Pharmacol. Physiol. 2007, 34, 135-141.

(23) Maumus, M.; Manferdini, C.; Toupet, K.; Peyrafitte, J. A.; Ferreira, R.; Facchini, A.; Gabusi, E.; Bourin, P.; Jorgensen, C.; Lisignoli, G.; Noel, D. Stem Cell Res. 2013, 11, 834-844.

(24) Simonaro, C. M.; Sachot, S.; Ge, Y.; He, X.; Deangelis, V. A.; Eliyahu, E.; Leong, D. J.; Sun, H. B.; Mason, J. B.; Haskins, M. E.; Richardson, D. W.; Schuchman, E. H. PLoS One 2013, 8, e62715. 